\title{
Reconciliación social como estrategia para la transformación de los conflictos sociopolíticos, variables asociadas e instrumentos de medición *
}

\section{Social Reconciliation as a Strategy for Transforming Socio-Political Conflicts, Associated Variables and Measurement Instruments}

\author{
Mónica Alzate \\ Instituto Tecnológico Metropolitano, Colombia \\ ORCID: http://orcid.org/0000-0002-9566-535X \\ Marcos Dono \\ Universidade de Santiago de Compostela, España
}

a Autor de correspondencia. Correo electrónico: monica.alzate.garcia@gmail.com

Para citar este artículo: Alzate, M., \& Dono, M. (2017). Reconciliación social como estrategia para la transformación de los conflictos sociopolíticos, variables asociadas e instrumentos de medición. Universitas Psychologica, 16(3), 1-10.

https://doi.org/ 10.11144/Javeriana.upsy16-3.rset

\section{RESUMEN}

La reconciliación social ha sido propuesta como una de las estrategias que permite la transformación de los conflictos y el restablecimiento de las relaciones intergrupales pacíficas. El objetivo de este trabajo es hacer una revisión sistemática de la reconciliación, las variables con las que se asocia y los instrumentos para medirla. La búsqueda se hizo en Psycinfo y en la Web of Science, y los resultados indican que el mayor porcentaje de artículos surge en los años 90 , las publicaciones abordan conflictos que se desarrollan a lo largo de todo el planeta: América, Europa, Oriente medio y África. Las variables con las cuales se relaciona estadística y teóricamente a la reconciliación se agrupan en cinco categorias: recuperación psicosocial, acercamiento de las partes confrontadas, resignificación endo y exogrupal, emociones y gestión del conflicto. Se registran 12 instrumentos con una fiabilidad adecuada para evaluar la reconciliación. Se concluye que con las cinco categorías de variables se podrían diseñar modelos explicativos y de diagnóstico que contribuyan a la promoción de los procesos de reconciliación social temprana, particularmente para aquellos paises que requieren transformar las dinámicas de confrontación violenta.

Palabras clave

dinámicas grupales; reconciliación; resolución de conflictos

\begin{abstract}
Social reconciliation has been posed as one of the strategies that allows for conflict transformation and re-establishment of peaceful intergroup relationships. The aim of this work is to carry out a systematic review of reconciliation, its associated variables and its measurement instruments. The search process was carried out using PsychInfo and Web of Science databases. Results show that the largest proportion of articles arised during the 90's, publications discuss conflicts developed all over the world: America, Europe, Middle East and Africa. The variables with which reconciliation is statistically and theoretically associated group within five categories: psychoscial recovery, rapprochement of confronted
\end{abstract}


parties, ingroup and outgroup re-signification, emotions and conflict management. Twelve adequately reliable measurements of reconciliation are registered. It is concluded that the five categories mentioned could be used to design explanatory and diagnostic models which could contribute to the promotion of early social reconciliation processes, specifically within those countries that require the transformation of violent confrontation dynamics.

Keywords

group dynamics; reconciliation; conflict resolution

Durante el siglo XXI, los grandes enfrentamientos internacionales, propios del siglo anterior, han dado paso a otro tipo de conflictos: los surgidos en el interior de los territorios. En los conflictos internos dos o más grupos que difieren en raza, religión, ideología, poder o privilegio se enfrentan violentamente para alcanzar la primacía sobre el otro o para eliminarlo (Staub, 2012), afectando de forma directa o indirecta a un amplio sector de la población de un país. Por ende, su transformación hacia una paz duradera requerirá de un cambio profundo en los individuos de los grupos enfrentados, así como modificaciones culturales e institucionales (Staub, 2005).

Esta transformación profunda es el proceso que se ha denominado reconciliación. Bar-Tal (2000) la define como 'la formación de una cultura de paz, resultado del cambio en las metas, objetivos y creencias acerca del propio grupo, el exogrupo, y las relaciones entre ambos'. La reconciliación se plantea así, como la forma en que las personas restablecen sus lazos sociales y mantienen la debida estabilidad en el territorio.

De la definición de Bar-Tal se deriva que la reconciliación implicará beneficios no solo al nivel de las relaciones entre las personas, sino también de los indicadores colectivos de bienestar social. Existe evidencia empírica para ambos niveles, el personal y el colectivo. Mukashema y Mullet (2012) hallaron una asociación significativa entre la reconciliación y la salud mental de las personas de los países que dejaron atrás un conflicto armado. Por su parte, Archick (2015) evidencia mejoras en la economía de Irlanda del Norte a lo largo del proceso de paz; entre 1997 y 2007 creció un $5.6 \%$ de media, crecimiento marginalmente mayor que el del resto del Reino Unido. En la misma franja temporal, el desempleo de Irlanda del Norte cayó de un $8.8 \%$ en 1997 a un $4.3 \%$ en 2007. Un ejemplo más lo encontramos en el progreso experimentado en Sierra Leona: el PIB aumentó, también el Índice de Desarrollo Humano, la inflación descendió y la deuda externa se redujo. Además, se promulgaron leyes para garantizar la igualdad de género y el desarrollo sostenible (Perrault, 2013).

Con base en los anteriores indicadores, se puede afirmar, cuanto menos, que la reconciliación es un proceso clave en la creación de una sociedad posconflictual con mejor salud mental y económicamente más estable. Pese a tales beneficios, estamos ante un tema con un muy limitado desarrollo teórico y empírico. De ahí que el objetivo del presente trabajo sea realizar una revisión sistemática sobre la reconciliación, las variables asociadas a esta y los instrumentos de medida utilizados para la evaluación del constructo.

\section{Método}

Diseño

Estudio descriptivo transversal de la producción científica sobre la reconciliación tras conflictos intergrupales que abarcan la esfera societal.

\section{Estrategia de búsqueda}

Se realizó una revisión sistemática en las bases de datos PsycInfo y WOS (Web of Science), utilizando los descriptores "reconciliation" and "intergroup conflict", para artículos científicos evaluados por expertos.

A partir de los títulos y resúmenes arrojados por las bases de datos, se dispuso la lectura del texto completo de aquellos documentos que cumplían con los criterios de inclusión. La selección de artículos requirió un acuerdo entre dos investigadores, que para el caso fue del $90 \%$. La lectura de los documentos completos incluyó 
la revisión de referencias bibliográficas, lo que permitió la búsqueda de fuentes secundarias.

\section{Criterios de selección}

A partir de la definición de reconciliación propuesta por Bar-Tal (2000), se establecieron los criterios de inclusión y de exclusión de artículos. Se determinó que los artículos incluidos en esta revisión debían referirse a procesos reconciliatorios de grandes grupos sociales como son los Estados nación. Por tanto, quedarían fuera aquellos trabajos que se centrasen en la reconciliación de las relaciones interpersonales (relaciones de pareja o familiares) o intergrupales que no alcanzan a involucrar a todos los miembros de una sociedad, tales como los conflictos laborales o entre bandas delictivas.

\section{Resultados}

Las bases de datos arrojan 146 documentos; el $5 \%$ de estos trabajos son anteriores al año 1992, resultado consistente con los aportes de Kelman (2008) quien plantea un incremento en los estudios de la reconciliación ante el aumento de los conflictos intraestatales que surgieron tras la culminación de la denominada "Guerra Fría".

Se seleccionaron 46 de las referencias para una lectura completa y lo mismo se hizo con 11 textos derivados de fuentes secundarias. De las 46 fueron incorporadas 28 a la revisión, al constatarse que cumplían los criterios de inclusión y exclusión; se mantuvieron todos los textos procedentes de fuentes secundarias.

Los artículos seleccionados abordan nueve conflictos: Colombia, Chile, Sudáfrica, Kenia, Ruanda, Oriente medio, Irlanda del norte, Alemania y Croacia (Tablas 1 a 3).

\section{TABLA 1}

Variables asociadas con la reconciliación intergrupal en Sudamérica

\begin{tabular}{lcl}
\hline Fuente & Conflicto & Variables \\
\hline $\begin{array}{l}\text { Alzate, Sabucedo y } \\
\text { Durán (2013) }\end{array}$ & Colombiano & $\begin{array}{l}\text { Confianza, actitud etnocéntrica, actitud negociadora, } \\
\text { legitimidad. }\end{array}$ \\
$\begin{array}{l}\text { Manzi y Gonzalez } \\
(2007)\end{array}$ & Chileno & $\begin{array}{l}\text { Ira, culpa, vergüenza, demanda de remordimiento, demanda } \\
\text { de verdad, victimización. }\end{array}$ \\
$\begin{array}{l}\text { Noor, Brown, Gonzalez, Chileno } \\
\text { Manzi y Lewis (2008) }\end{array}$ & $\begin{array}{l}\text { Identificación endogrupal, identificación endogrupal común, } \\
\text { empatia, confianza, victimización competitiva, perdón. }\end{array}$ \\
\hline
\end{tabular}

Fuente: elaboración propia.

TABLA 2

Variables asociadas con la reconciliación intergrupal en África y Oriente Medio

\begin{tabular}{|c|c|c|}
\hline Fuente & Conflicto & Variables \\
\hline Chapman (2007) & Apartheid sudafricano & Verdad y memoria colectiva \\
\hline Gibson (2004) & Apartheid sudafricano & Verdad y memoria colectiva \\
\hline $\begin{array}{l}\text { Kamau Giner-Sorolla y } \\
\text { Zeven (2013) }\end{array}$ & $\begin{array}{l}\text { Colonización británica en } \\
\text { Kenia }\end{array}$ & $\begin{array}{l}\text { Expresiones de culpa o de vergüenza en el } \\
\text { contexto de disculpas oficiales de un grupo } \\
\text { perpetrador. }\end{array}$ \\
\hline $\begin{array}{l}\text { Mukashema y Mullet } \\
\text { (2012) }\end{array}$ & $\begin{array}{l}\text { Conflicto entre Hutus y } \\
\text { Tutsis en Ruanda }\end{array}$ & Perdón \\
\hline Nadler y Liviatan (2006) & Palestino-israeli & $\begin{array}{l}\text { Expresiones de empatia, expresiones de } \\
\text { responsabilidad, confianza. }\end{array}$ \\
\hline $\begin{array}{l}\text { Shnabel, Nadler, Ullrich, } \\
\text { Dovidio y Carmi (2009) }\end{array}$ & $\begin{array}{l}\text { Palestino-israeli } \\
\text { Germano-judio }\end{array}$ & $\begin{array}{l}\text { Modelo de reconciliación basado en las } \\
\text { necesidades: necesidad de aceptación } \\
\text { social en perpetradores, necesidad } \\
\text { empoderamiento en victimas. }\end{array}$ \\
\hline $\begin{array}{l}\text { Shnabel, Halabi y Noor } \\
\text { (2013) }\end{array}$ & Palestino-israeli & Intervenciones de recategorización. \\
\hline $\begin{array}{l}\text { Shnabel, Nadler y } \\
\text { Dovidio (2014) }\end{array}$ & Palestino-israeli & $\begin{array}{l}\text { Empoderamiento, aceptación social, } \\
\text { confianza. }\end{array}$ \\
\hline Staub (2005) & $\begin{array}{l}\text { Conflicto entre Hutus y } \\
\text { Tutsis en Ruanda }\end{array}$ & $\begin{array}{l}\text { Entrenamiento especializado de } \\
\text { mediadores comunitarios de agencias pro } \\
\text { reconciliación. }\end{array}$ \\
\hline $\begin{array}{l}\text { SimanTov-Nachlieli y } \\
\text { Shnabel (2013) }\end{array}$ & Palestino-israeli & Empoderamiento, aceplación social. \\
\hline
\end{tabular}

Fuente: elaboración propia.

TABLA 3

Variables relacionadas con la reconciliación intergrupal en Europa

\begin{tabular}{|c|c|c|}
\hline Fuente & Conflicto & Variables \\
\hline Kosić et al. (2011) & $\begin{array}{l}\text { Norirlandés } \\
\text { Serbo-croata }\end{array}$ & 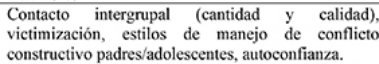 \\
\hline $\begin{array}{l}\text { Kosić y Livi (2012) } \\
\text { Noor, Brown y } \\
\text { Prentice (2008a) }\end{array}$ & $\begin{array}{l}\text { Serbo-croata } \\
\text { Norirlandés }\end{array}$ & $\begin{array}{l}\text { Estilos de comunicación en la familia, victimización, } \\
\text { modelo orientado a la reconciliación: victimización } \\
\text { competitiva, identificación endogrupal, confianza, } \\
\text { empatia. }\end{array}$ \\
\hline Noor et al. (2008) & Norirlandés & $\begin{array}{l}\text { Identificación endogrupal, identificación endogrupal } \\
\text { común, empatia, confianza, victimización } \\
\text { competitiva, perdón. }\end{array}$ \\
\hline Shnabel et al. (2009) & $\begin{array}{l}\text { Conflicto germano- } \\
\text { judio }\end{array}$ & $\begin{array}{l}\text { Modelo basado en las necesidades: necesidad de } \\
\text { aceptación perpetradores, necesidad empoderamiento } \\
\text { victimas. }\end{array}$ \\
\hline Tam et al. (2007) & Norirlandés & $\begin{array}{l}\text { Ira, deshumanización, actitudes positivas hacia el } \\
\text { exogrupo, contacto. }\end{array}$ \\
\hline Tam et al. (2008) & Norirlandés & $\begin{array}{l}\text { Deshumanización, empatia, ira, miedo, emociones } \\
\text { positivas, contacto intergrupal. }\end{array}$ \\
\hline
\end{tabular}

Fuente: elaboración propia.

\section{Variables asociadas a la reconciliación}

A partir de la lectura de los manuscritos se conformaron cinco categorías de variables: a) procesos asociados a la recuperación psicosocial; b) procesos asociados al acercamiento de las partes; c) resignificación grupal; d) variables 
emocionales y e) estrategias de gestión del conflicto. Cada una de las categorías emerge de la relación teórica o empírica entre las diversas variables descritas por los autores de los manuscritos seleccionados (Figura 1).

\section{Figura 1.}

Reconciliación social y categorías asociadas.

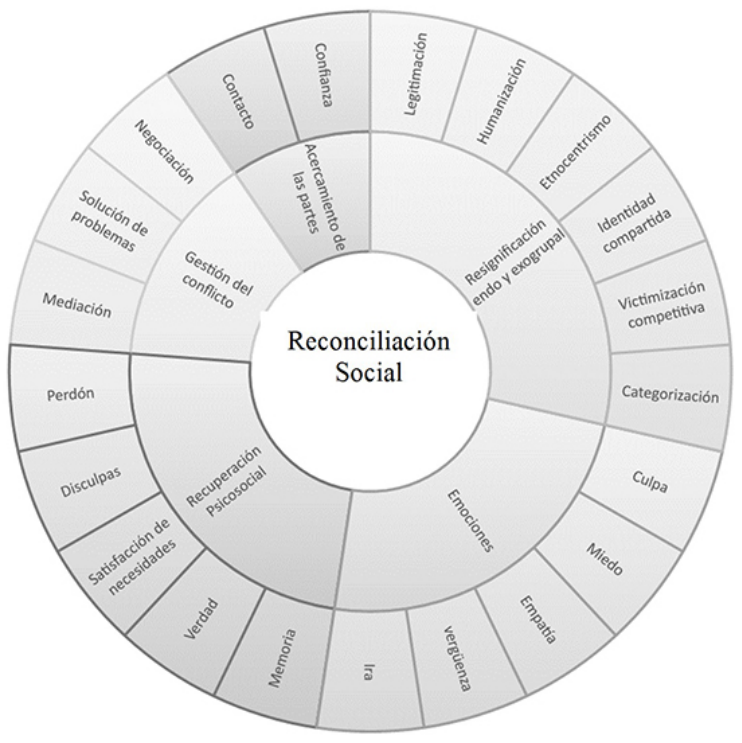

Fuente: elaboración propia.

\section{Procesos asociados a la recuperación psicosocial}

En esta primera categoría se han incluido variables que permiten a los individuos y a los grupos recuperarse del daño sufrido: el perdón, las disculpas, la satisfacción de necesidades identitarias, la verdad y la memoria. A continuación, se presenta el tipo de relación que establece cada una de estas variables con la reconciliación.

La variable que se encuentra más estrechamente asociada a la reconciliación, es el perdón. Incluso algunos autores afirman que puede ser considerado como un aspecto de esta (Staub, 2012); para otros, tiene un rol predictor de la reconciliación (Noor et al., 2008a) o es un paso previo de esta (Kadiangandu \& Mullet, 2007). Ambas variables son concebidas como constructos diferentes, pero con una enorme proximidad.
Asociada al perdón está la petición de disculpas, una variable que también fue encontrada como buena predictora de la reconciliación (Mukashema \& Mullet, 2012; Shnabel et al., 2009) y puede mostrar diferente eficacia según el grado de culpabilidad asignado al grupo agresor. La investigación de Kamau et al. (2013) indica que si la culpabilidad percibida es alta, la respuesta mejor evaluada por el grupo agredido es la que manifiesta vergüenza por parte del agresor; cuando la culpabilidad asignada al grupo agresor es baja, la mera expresión de culpa será más eficaz que la vergüenza.

Dentro de este ciclo de "disculpas-perdón", aparece la satisfacción de necesidades identitarias asociada a la reconciliación. Según Nadler y Shnabel (2008) las personas participantes del conflicto asumen identidades colectivas de víctimas o perpetradores y esto genera un tipo de necesidad que es específica para cada grupo, de cara al proceso reconciliatorio; quienes se identifican como víctimas tendrán una mayor necesidad de verse empoderadas y quienes se reconocen como perpetradores tendrán una mayor necesidad de ser aceptados socialmente. Experimentalmente, se observó que los mensajes de empoderamiento y aceptación favorecen la reconciliación cuando proceden de la otra parte implicada en el conflicto, pero fueron ineficaces cuando proceden de una tercera parte neutral (Shnabel et al., 2014).

El abordar los ciclos de disculpas y perdón nos lleva a las Comisiones de la Verdad y la Reconciliación (CVR), pues estas constituyen una de las formas de intervención colectiva más utilizada en el marco de la reconciliación intergrupal. Para Kelman (2008), confrontar la historia y acordar la verdad es un componente esencial de cualquier esfuerzo reconciliatorio, y Staub (2013) afirma que una memoria colectiva más verídica puede ayudar a los grupos a tomar conciencia y responsabilidad de sus acciones, así como a aceptar una historia compartida.

Con un enfoque más empírico, Gibson (2004) evaluó las consecuencias de la CVR en Sudáfrica, encontrando correlaciones positivas entre la aceptación de la verdad propuesta por la CVR y la actitud reconciliatoria en todos los grupos étnicos 
participantes. Sin embargo, no todos los trabajos examinados constatan los procesos de memoria histórica como positivos para la reconciliación. Así, Chapman (2007) evaluó esta misma CVR en Sudáfrica y, tras realizar una encuesta a los participantes de dicho proceso, concluyó que no favoreció de forma relevante la reconciliación intergrupal.

\section{Procesos asociados al acercamiento de las partes}

Esta segunda categoría de variables asociadas a la reconciliación está conformada por la confianza y el contacto intergrupal. La confianza es definida por Kollock (1994) como 'un mecanismo psicológico cuyo objetivo es sobreponerse a situaciones sociales inciertas a

través de asunciones benignas del comportamiento de otros'. En este sentido, BarTal (2000) la considera como un factor inherente al proceso de reconciliación y Baron (2008) la concibe como un factor que incrementa la eficacia de los acercamientos con objetivos de reconciliación.

En esta revisión sistemática, se encontraron diversos trabajos que utilizan modelos de ecuaciones estructurales y que han buscado establecer el papel de la confianza sobre la reconciliación, en algunos casos fue considerada como una variable mediadora y en otros se observa una cierta capacidad predictiva sin mediar ni ser mediada por otras variables. Noor et al. (2008a) la consideran mediadora de la relación entre el perdón y la reconciliación; Freeman (2012) postula que puede facilitar la reconciliación terciando el aumento de empatía hacia el exogrupo y la disminución de estereotipia y de prejuicio. Por otra parte, el trabajo de Alzate et al. (2013) evidencia que la confianza puede llegar a tener una doble relación con la reconciliación, tanto matizada por otras variables como directa sobre la reconciliación.

La confianza es una variable que frecuentemente se relaciona con el contacto intergrupal, y este a su vez se relaciona con los procesos reconciliatorios (Hewstone et al., 2008). En un estudio realizado con jóvenes norirlandeses se analizó la calidad y la cantidad del contacto intergrupal; la primera variable emergió como predictora positiva de la reconciliación, cosa que no ocurrió con la cantidad de contacto (Kosić, Noor, \& Mannetti, 2012). También se ha encontrado que el contacto entre jóvenes palestinos e israelíes supuso un incremento en las actitudes positivas hacia el exogrupo, incluso después de haber pasado un año (Staub, 2013).

Los efectos del contacto pueden analizarse en tres momentos: el preconflicto ha recibido muy poca atención, sin embargo, las hipótesis apuntan a que facilitaría una posterior reconciliación; el contacto tras el conflicto es el más estudiado y también ha probado ser eficaz para la reducción de prejuicios y la promoción de confianza y perdón hacia el exogrupo y el contacto durante el conflicto, no presenta datos para avalar o descartar su eficacia en la promoción de la reconciliación (Wagner \& Hewstone, 2012).

Autores como Dovidio (2008) o Riek et al. (2008) proponen que el contacto induce al cambio en la categorización social de los grupos y, según Noor, Brown y Prentice (2008b) esto promueve una identidad grupal más inclusiva que reduce las actitudes negativas hacia el exogrupo. De esto se desprende la conformación de la próxima categoría.

\section{Resignificación grupal}

En el proceso de categorización social los individuos se clasifican a sí mismos en una categoría social y fuera de otras, que no es nociva por sí misma, sin embargo, dentro de una situación de conflicto puede llevar a la generación de una imagen distorsionada del propio grupo y del grupo adversario. La eliminación de dichas categorías y la promoción de una nueva identidad ayuda a eliminar las barreras que dificultan la reconciliación (JanoffBulman \& Werther, 2008). Shnabel et al. (2013) utilizaron intervenciones de recategorización y las sometieron a comprobación empírica en el contexto del conflicto israelí-palestino; la recategorización fue efectiva para el perdón y la reconciliación cuando se establecieron categorías 
de víctimas y perpetradores, pero no ocurrió lo mismo cuando se ponían en oposición las categorías de la pertenencia regional.

Relativo a la categorización como víctima de un conflicto cabe mencionar la relación inversa entre la reconciliación y la victimización competitiva, entendida esta última como 'los esfuerzos de ambos grupos en conflicto para reivindicar que el propio grupo ha sufrido más que la otra parte' (Noor et al., 2008; Noor et al., 2008a). La victimización competitiva activa la identificación endogrupal con la categoría a la que se pertenece, y esta se asocia negativamente con el perdón y con la reconciliación; mientras que la identificación con una categoría endogrupal compartida por los dos grupos se relaciona positivamente con dichos procesos (Noor et al., 2008).

Los procesos de categorización implican la conformación de una imagen sobre el propio grupo y sobre los "otros", que será menos positiva bajo condiciones de conflicto. Janoff-Bulman y Werther (2008) afirman que, en condiciones de conflicto, se hará una atribución automática de inmoralidad y malas intenciones hacia la otra parte que es simultánea a la atribución de moralidad y buenas intenciones al endogrupo, esto es lo que se conoce como etnocentrismo. Miron y Branscomben (2008) sostienen que en condiciones de conflicto los miembros del endogrupo van a ser menos proclives a humanizar a los miembros del exogrupo.

Por otra parte, para no ver dañada su imagen, los grupos considerados como perpetradores tienden a distanciarse social y emocionalmente de las víctimas y lo hacen a través de la deslegitimación (Nadler \& Shnabel, 2008). El proceso opuesto, la legitimación, predice positivamente la reconciliación (Alzate et al., 2013).

\section{Variables emocionales}

Una cuarta categoría de variables está conformada por emociones positivas y negativas asociadas a la reconciliación. La emoción positiva más recurrente es la empatía, definida como 'la capacidad de ponerse en el lugar del otro y apreciar su forma de sentir' (Noor et al., 2008). En cuanto a emociones negativas, se encuentran el miedo y la ira; la primera predice conductas evasivas, mientras que la ira predice conductas agresivas hacia el exogrupo (Devos, Silver, Mackie, \& Smith, 2002). Ambas emociones dificultan el proceso reconciliatorio pues actúan como predictores negativos del perdón (Tam et al., 2008). Otras emociones negativas examinadas por Manzi y Gonzalez (2007) son la culpa y la vergüenza colectiva; la primera se relacionó positivamente con el perdón y la reparación en ambos grupos (víctimas y perpetradores). La vergüenza ofreció resultados menos consistentes, se relacionó positiva y significativamente con la reparación para el grupo perpetrador y con el perdón en el grupo de víctimas. En esta misma línea van los resultados de Noor et al. (2008b).

\section{Estrategias de gestión del conflicto}

Esta categoría de variables agrupa las actitudes y habilidades de las personas para afrontar los conflictos. En este sentido, se observa que la actitud negociadora hace más probable la disposición hacia la reconciliación (Alzate et al., 2013), un resultado semejante a lo observado en jóvenes con un estilo constructivo de solución de conflictos (Kosić \& Livi, 2012; Kosić et al., 2012).

Una última variable por mencionar es el entrenamiento de mediadores comunitarios: se observó una mejor actitud reconciliadora de los grupos acompañados por personas que participaron en un programa de entrenamiento para mediadores (Staub, 2005).

\section{Instrumentos de medida para la reconciliación social}

El segundo objetivo de esta revisión consiste en dar cuenta de los instrumentos utilizados para medir la reconciliación. Se encontraron 12 cuestionarios recogidos en la Tabla 4. $\mathrm{Si}$ bien se observan diferencias en las definiciones operacionales de la reconciliación, 
estas discrepancias guardan congruencia con las dos concepciones de reconciliación propuestas por Nadler y Shnabel (2008), la instrumental y la socioemocional, los 12 instrumentos de medición podrían agruparse en alguna de estas dos dimensiones. Los cuestionarios muestran valores de alpha entre 0.68 y 0.95 , esto indica un buen nivel de fiabilidad. La mayoría utilizan preguntas que se responden con escalas tipo Likert (Tabla 4).

\section{TABLA 4}

Instrumentos de medida de reconciliación

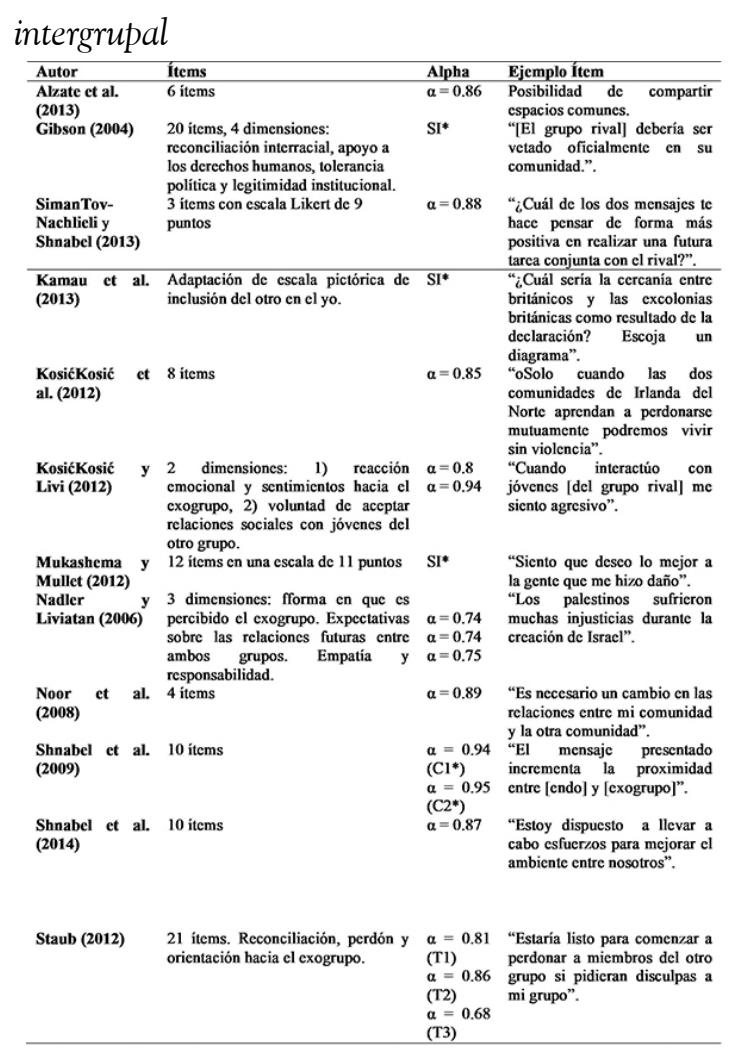

Nota. SI: sin información; C1 representa la condición donde el mensaje es de empoderamiento; C2 representa la condición donde el mensaje es de aceptación. Fuente: elaboración propia.

\section{Conclusiones}

En este trabajo, se confirma el planteamiento de Kelman (2008) para quien la reconciliación es una estrategia que tiene su mayor expresión a partir de los años 90, de ahí que el desarrollo teórico de la reconciliación sea reciente. Aun así, en la presente sistematización se pone en evidencia que estas décadas han bastado para que surjan múltiples perspectivas sobre el constructo.

A partir de los resultados, se puede afirmar que la diferente procedencia de las investigaciones ha enriquecido la caracterización del tipo de reconciliación que se vive en cada uno de los países estudiados; sin embrago, esto también ha complejizado el establecimiento de consensos entre los investigadores. Con esta investigación se espera haber contribuido a la sistematización de categorías que faciliten la identificación de resultados comunes a diversos estudios y la conformación de nuevos modelos explicativos y/o de intervención que permitan cambiar las dinámicas de violencia. La integración de algunas de las 21 variables que en esta revisión se asocian a los procesos reconciliatorios bien podrían facilitar los procesos de pacificación.

El segundo objetivo del presente manuscrito era encontrar escalas que evaluasen la reconciliación, sobre estas se puede concluir que las medidas fueron creadas ad hoc para cada contexto nacional, tan solo algunos trabajos como el de Noor et al. (2008) y el de Mukashema y Mullet (2012) hacen un esfuerzo por la validación ecológica de sus hallazgos entre diferentes países, lo que permite ver una futura línea de investigación que busque la validación de instrumentos transculturales. Por otra parte, una alta proporción de los instrumentos sobre reconciliación fueron aplicados a estudiantes universitarios, de ahí la pertinencia de ampliar las investigaciones con muestras representativas de la población general y de realizar estudios longitudinales que avalen la estabilidad de los hallazgos. Valga señalar que aunque los trabajos no sean longitudinales, sí se han encontrado coincidencias en contextos culturalmente diferentes, en variables tan relevantes como la victimización competitiva, la confianza, el papel del perdón y de emociones positivas, entre otros.

Una de las limitantes de esta revisión es su predominio de contenidos psicológicos, por tanto, sería interesante emprender nuevas revisiones con una perspectiva más multidisciplinar. Pues, aunque el contenido 
psicosocial de la reconciliación es innegable, puede conducir a una visión "sobrepsicologizada” que, según Rouhana (2011), podría no ser eficaz para el proceso de pacificación. Por otro lado, la mera revisión de la bibliografía científica no permite visibilizar el esfuerzo de distintas organizaciones sociales que a través de su trabajo consiguen hacer de la reconciliación una realidad tangible. A pesar de las limitaciones antes mencionadas, los procesos de reconciliación social son una necesidad innegable para países que enfrentan conflictos violentos de larga duración. Esta revisión sistemática puede proveer antecedentes útiles para los investigadores sociales interesados en desarrollar esta temática.

\section{Agradecimientos}

Financiado por la Consellería de Cultura, Educación e Ordenación Universitaria de la Xunta de Galicia. Referencia: ED431B 2016/017.

\section{Referencias}

Alzate, M., Sabucedo, J. M., \& Durán, M. (2013). Antecedents of the attitude towards intergroup reconciliation in a setting of armed conflict. Psicothema, 25(1), 61-66. http:// doi.org/10.7334/psicothema2011.390

Archick, K. (2015). Northern Ireland: The Peace Process (CRS Report RS21333). Washington, DC: Congressional Research Service.

Baron, R. (2008). Reconciliation, trust, and cooperation: Using bottom-up and topdown strategies to achieve peace in the Israeli-Palestinian conflict. En A. Nadler, T. Malloy \& J. D. Fisher (Eds.), The social psychology of intergroup reconciliation (pp. 275-298). Oxford: Oxford University Press.

Bar-Tal, D. (2000). From intractable conflict through conflict resolution to reconciliation: Psychological analysis. Political Psychology, 21 (2), 351-365. http:// doi.org/10.1111/0162-895X.00192
Chapman, A. R. (2007). Truth commissions and intergroup forgiveness: The case of the South African Truth and Reconciliation Commission. Peace and Conflict: Journal of Peace Psychology, 13(1), 51-69. http:// doi.org/10.1037/h0094024

Devos, T., Silver, L. A., Mackie, D. M., \& Smith, E. R. (2002). Experiencing intergroup emotions. En D. M. Mackie \& E. R. Smith (Eds.), From prejudice to intergroup emotions (pp. 111-134). Nueva York: Taylor $\&$ Francis.

Dovidio, J. F. (2008). Majority and minority perspectives in intergroup relations: The role of contact, group representations, threat, and trust in intergroup conflict and reconciliation. En A. Nadler, T. Malloy \& J. D. Fisher (Eds.), The social psychology of intergroup reconciliation (pp. 227-253). Oxford: Oxford University Press.

Freeman, C. M. L. (2012). The psychosocial need for intergroup contact: Practical suggestions reconciliation initiatives in Bosnia and Herzegovina and beyond. Intervention, 10(1), 17-29.

Gibson, J. L. (2004). Overcoming Apartheid: Can truth reconcile a divided nation? Politikon, 31(2), 129-155. http:// doi.org/10.1080/0258934042000280698

Hewstone, M., Kenworthy, J. B., Cairns, E., Tauch, N., Hughes, J., Tam, T., Voci, A.,... Pinder, C. (2008). Stepping stones to reconciliation in Northern Ireland: Intergroup contact, forgiveness, and trust. En A. Nadler, T. Malloy \& J. D. Fisher (Eds.), The social psychology of intergroup reconciliation (pp. 119-227). Oxford: Oxford University Press. http://doi.org/10.1093/ acprof:oso/9780195300314.003.0010

Janoff-Bulman, R., \& Werther, A. (2008). The social psychology of respect: Implications for delegitimization and reconciliation. En A. Nadler, T. Malloy \& J. D. Fisher (Eds.), The social psychology of intergroup reconciliation (pp. 145-170). Oxford: Oxford University Press. 
Kadiangandu, J. K., \& Mullet, E. (2007). Intergroup forgiveness: A Congolese perspective. Peace and Conflict: Journal of Peace Psychology, 13(1), 37-49. http:// doi.org/10.1037/h0094023

Kamau, C., Giner-Sorolla, R., \& Zeven, S. (2013). Reconciliation responses, blame, and expressions of guilt or shame. Journal of Applied Social Psychology, 43, 287-292. http://doi.org/10.1111/jasp.12048

Kelman, H. C. (2008). Reconciliation from a social-psychological perspective. En A. Nadler, T. Malloy \& J. D. Fisher (Eds.), The social psychology of intergroup reconciliation (pp. 15-32). Oxford: Oxford University Press.

Kollock, P. (1994). The emergence of exchange structures: An experimental study of uncertainty, commitment, and trust. American Journal of Sociology, 100(2), 313-345.

Kosić, A., \& Livi, S. (2012). Study of perceived parental communication and propensity towards reconciliation among youth in Vukovar (Croatia). Journal on Ethnopolitics and Minority Issues in Europe, 11 (4), 51-80.

Kosić, A., Noor, M., \& Mannetti, L. (2012). The propensity toward reconciliation among young people in Northern Ireland and Croatia: The role of conflict management styles within the family. Group Processes $\mathbb{E}$ Intergroup Relations, 15(1), 3-19.

Manzi, J., \& Gonzalez, R. (2007). Forgiveness and reparation in Chile: The role of cognitive and emotional intergroup antecedents. Peace and Conflict: Journal of Peace Psychology, 13(1), 71-91. http:// doi.org/10.1080/10781910709336769

Miron, A. M., \& Branscomben, N. R. (2008). Social categorization, standards of justice, and collective guilt. En A. Nadler, T. Malloy $\&$ J. D. Fisher (Eds.), The social psychology of intergroup reconciliation (pp. 77-96). Oxford: Oxford University Press.

Mukashema, I., \& Mullet, E. (2012). Unconditional forgiveness, reconciliation sentiment, and mental health among genocide victims in Rwanda. Social
Indicators Research, 113, 121-132. http:// doi.org/10.1007/s11205-012-0085-x

Nadler, A., \& Shnabel, N. (2008). Instrumental and socioemotional paths to intergroup reconciliation and the Needs-Based Model of Socioemotional Reconciliation. En A. Nadler, T. Malloy \& J. D. Fisher (Eds.), The social psychology of intergroup reconciliation (pp. 37-56). Oxford: Oxford University Press. http://doi.org/10.1093/ acprof:oso/9780195300314.003.0003

Noor, M., Brown, R. J., Gonzalez, R., Manzi, J., \& Lewis, C. A. (2008). On positive psychological outcomes: What helps groups with a history of conflict to forgive and reconcile with each other? Personality and Social Psychology Bulletin, 34(6), 819-832. http://doi.org/10.1177/0146167208315555

Noor, M., Brown, R. J., \& Prentice, G. (2008a). Precursors and mediators of intergroup reconciliation in Northern Ireland: A new model. British Journal of Social Psychology, 47(3), 481-495.

Noor, M., Brown, R. J., \& Prentice, G. (2008b). Prospects for intergroup reconciliation: Social-psychological predictors of intergroup forgiveness and reparation in Northern Ireland and Chile. En A. Nadler, T. Malloy \& J. D. Fisher (Eds.), The social psychology of intergroup reconciliation (pp. 97-114). Oxford: Oxford University Press.

Perrault, F. (2013). Sierra Leone. Country Strategy Paper 2013-2017. Costa de Marfil: African Development Bank Group.

Riek, B. M., Gaertner, S. L., Dovidio, J. F., Brewer, M. B., Mania, E. W., \& Lamoreaux, M. J. (2008). A social-psychological approach to postconflict reconciliation. En A. Nadler, T. Malloy \& J. D. Fisher (Eds.), The social psychology of intergroup reconciliation (pp. 255-273). Oxford: Oxford University Press.

Rouhana, N. N. (2011). Key issues in reconciliation: Challenging traditional assumptions on conflict resolution and power dynamics. En D. Bar-Tal (Ed.), Intergroup conflicts and their resolution: $\mathrm{A}$ social psychological perspective (pp. 291-314). Nueva York: Psychology Press. 
Shnabel, N., Halabi, S., \& Noor, M. (2013). Overcoming competitive victimhood and facilitating forgiveness through recategorization into a common victim or perpetrator identity. Journal of Experimental Social Psychology, 49(5), 867-877. http:// doi.org/10.1016/j.jesp.2013.04.007

Shnabel, N., Nadler, A., \& Dovidio, J. F. (2014). Beyond need satisfaction: Empowering and accepting messages from third parties ineffectively restore trust and consequent reconciliation. European Journal of Social Psychology, 44(2), 126-140.

Shnabel, N., Nadler, A., Ullrich, J., Dovidio, J. F., \& Carmi, D. (2009). Promoting reconciliation through the satisfaction of the emotional needs of victimized and perpetrating group members: The needs-based model of reconciliation. Personality and Social Psychology Bulletin, 35(8), 1021-1030. http://doi.org/10.1177/0146167209336610

Staub, E. (2005). Healing, reconciliation, forgiving and the prevention of violence after genocide or mass killing: An intervention and its experimental evaluation in Rwanda. Journal of Social and Clinical Psychology, 24(3), 297-334.

Staub, E. (2012). Genocide, mass killing, and violent conflict: Prevention and reconciliation. En L. R. Tropp (Ed.), The Oxford handbook of intergroup conflict (pp. 273-290). Nueva York: Oxford University Press.

Staub, E. (2013). Building a peaceful society: Origins, prevention, and reconciliation after genocide and other group violence. American Psychologist, 68(7), 576-589. http://doi.org/10.1037/a0032045

Tam, T., Hewstone, M., Kenworthy, J. B., Cairns, E., Marinetti, C., Geddes, L., \& Parkinson, B. (2008). Postconflict reconciliation: Intergroup forgiveness and implicit biases in Northern Ireland. Journal of Social Issues, 64(2), 303-320. http:// doi.org/10.1177/1368430207071345

Wagner, U., \& Hewstone, M. (2012). Intergroup contact. En L. R. Tropp (Ed.), The
Oxford handbook of intergroup conflict (pp. 193-209). Nueva York: Oxford University.

\section{Notas}

* Artículo de revisión. 